\title{
Fun outreach in Optics: Physics League
}

\section{Divulgación en Óptica divertida: Physics League}

\author{
V. Gonzalez-Fernandez ${ }^{1,2,5}$, F. Heviaa ${ }^{1,3}$, B. G. del Rio ${ }^{1,2}$, L. Sanchez-Tejerina ${ }^{1,4}$ and \\ D. Mateos ${ }^{1,2^{*}}$ \\ 1. Asociación sin ánimo de lucro Physics League, BS005, Facultad de Ciencias, Paseo de Belén 7, CP 47011, \\ Valladolid, España \\ 2. Dpto. de Física Teórica, Atómica y Óptica, Facultad de Ciencias, Universidad de Valladolid, Paseo de Belén \\ 7, CP 47011, Valladolid, España \\ 3. Dpto. de Física Aplicada, Facultad de Ciencias, Universidad de Valladolid, Paseo de Belén 7, CP 47011, \\ Valladolid, España \\ 4 Dpto. de Electricidad y Electrónica, Universidad de Valladolid, Paseo de Belén 7, CP 47011, Valladolid, \\ España.
}

${ }_{(*)}$ E-mail: mateos@goa.uva.es

Received: $11 / 12 / 2019$

DOI: 10.7149/OPA.53.1.51036
S: SEDOPTICA member

Accepted: 14/02/2020 


\section{REFERENCES AND LINKS / REFERENCIAS Y ENLACES}

[1] M. Artime, A. Martínez, J. Peláez (2020): Naukas. Available on: https://naukas.com/ [Consulta: 6 de febrero de 2020].

[2] J. Santaolalla (2020): Date un Vlog. Available from: https://www.youtube.com/channel/UCQX_MZRCaluNKxkywkLEgfA [viewed 6th of February, 2020].

[3] J. L. Crespo (2020): QuantumFracture. Available from: https://www.youtube.com/user/QuantumFracture [viewed 6th of February, 2020].

[4] Fundación Española para la Ciencia y la Tecnología, British Council (2020): Famelab. Talking Science. Available from: https://www.famelab.es/ [viewed 6th of February, 2020].

[5] Pint of Science España (2020). Pint of Science España. Available from: https://pintofscience.es/ [viewed 6th of February, 2020].

[6] Ciencia en Acción (2015) Premiados Ciencia en Acción 2015. Available from: http://anteriores.cienciaenaccion.org/2015/es/2015/index-premiados.html [viewed 6th of February, 2020].

[7] EPS Young Minds Project (2020): Young Minds Best Activity Award. Available from: https://www.epsyoungminds.org/category/activity/ym-best-activity/ [viewed 6th of February, 2020].

[8] P. A. Tipler, G. Mosca, Física para la ciencia y la tecnología, 6th edition. Reverté (2010).

[9] E. Hecht, Óptica, 5th edition Pearson Educación (2016).

[10] J. Casas, Óptica, 7th edition. Librería PONS (1994).

[11] Asociación Physics League (2015): Game of Physics: Season 1 [online video]. Available from: https://www.youtube.com/watch?v=6qAW4PhUmmE [viewed 6th of February, 2020].

[12] Asociación Physics League (2016): Other Worlds' Physics [online video]. Available from: https://www.youtube.com/watch?v=TPkmV4biz9s [viewed 6th of February, 2020].

[13] J. M. Williams, A. Lit, Luminance-dependent visual latency for the Hess effect, the Pulfrich effect, and simple reaction time. Vision Res. 23(2), 171-799 (1983).

[14] Interesting engineering, Inc. (2020): The Pythagorean Cup - The Cup That Spills Your Drink When You Get Too Greedy. Available from: https://interestingengineering.com/the-pythagorean-cup-the-cupthat-spills-your-drink-when-you-get-too-greedy [viewed 6th of February, 2020].

[15] Asociación Physics League (2016): Scary Physics English Version [online video]. Available from: https://www.youtube.com/watch?v=XT_S_SL4EOI [viewed 6th of February, 2020].

[16] J. Steinmeyer, The Science Behind the Ghost: A Brief History of Pepper's Ghost, The Victorian Theatrical Sensation. Hahne (2013)

\section{Introduction}

In recent years, scientific outreach has gained much attention among the scientific community in an effort to create a more informed and inquisitive society on science related matters such as vaccines, global warming, and clean energy resources. Over time, outreach skills have become more valued among the various capabilities of researchers. In the internet era, most of the dissemination has been addressed using recent tools such as blogs, like 'Naukas' [1], a popular science blog for the general public, where several scientist from different fields and journalists periodically post their contributions in an effort to bring science closer to a general public who a priori has no scientific training. In addition to science blogs, podcasts have also become very popular, and YouTube channels entirely dedicated to scientific dissemination have appeared. On this latter category, some channels have reached more than one million subscribers, for instance 'Date un Vlog' [2] (Javier Santaolalla, physicist), whose videos are devoted mainly to Astronomy and Particle Physics, or 'QuantumFracture' [3] (José Luis Crespo, physicist), whose videos deal primarily with Quantum Physics. 
All these media have shown that there is a target audience for whom science is a subject of interest. The challenge in many cases is not only to know how to connect with that audience, but also how to gain attention from a less interested public. Thus, we have seen the birth of several initiatives, such as the 'Famelab' [4] competition, where researchers present their work in the form of humorous monologues, or 'Pint of Science' [5], where scientists give simplified and informal lectures about their research field.

The non-profit association Physics League was born in December 2014 at the heart of the Physics Section of the Faculty of Sciences of the University of Valladolid, and immediately started incorporating students from both the Degree and the existing Master as well as Ph.D. students. At present, the association has 77 members, and since its beginnings, it has managed to reach an audience of almost 20,000 people, apart from the potential impact due to its multiple appearances in regional and national television and social media. The activities carried out have led Physics League to earn several international prizes, such as the First Prize in the 'Physics in Society' [6] category in the 'Science in Action XVI' (2015) competition, the 'EPL 2015 Best Young Minds Activity Award' [7], given by the Young Minds project of the European Physical Society (EPS) and Europhysics Letters (EPL), and the 'OSA Excellence Prize Grand Prize for Community/Youth Education Outreach', awarded in 2017 by the American Optical Society (OSA).

One of the strengths of Physics League's approach is the use of live, interactive, and experimental demonstrations, as the audience has commented on several occasions regardless of the type of public. In the specific case of teenage students, it is also a very good complement to the traditional science education in schools, characterized generally by a lack of laboratory work. Since its inception, Physics League has opted for an informal approach, setting most of its activities in television series, such as Game of Thrones, or famous films, like Pirates of the Caribbean or classical horror films. These settings, along with the theatrical productions, have proved to be a very successful way of attracting the attention of both the youngest and adolescent audiences, as well as of the most adult attendees who come to our performances.

Here we present a summary of the contents addressed in those Physics League's activities related to Optics and Photonics, including: small and large workshops, educative stage plays, and a small escape room. All these contents have been used in more than 100 activities since December 2014.

\section{Projects and activities}

Throughout these years, the association has designed and carried out different shows and workshops for a diversity of audiences. All these activities are characterized by a very direct interaction with the public, who also perform and interact with the experiments themselves. In this section we will detail all those activities whose main theme is Optics and Photonics. All the explanations of the physical phenomena detailed here can be found in the usual bibliography for undergraduate Physics courses [8-10], and therefore they will not be reproduced here to avoid being repetitive.

\section{2.a. Game of Physics: House Baratheon}

This workshop is one third of a larger workshop called 'Game of Physics' [11], set on the television series 'Game of Thrones', where three areas of Physics are addressed: 1) Thermodynamics, set with the House Targaryen, 2) Electromagnetism, associated with the White Walkers, and 3) Optics, linked to the Lord of Light, R'hllor.

This workshop, aimed at students in their final years of secondary school, is designed as an introduction to some essential concepts in Optics: Geometrical Optics, light-matter interaction, luminescence, etc.

Geometrical optics experiments are carried out with an optics kit (Ventus), equipped with different optical materials: laser, convergent and divergent lenses, plain and convex mirrors, and a glass tube to illustrate total reflection in an optical fibre. In addition, schemes of several optical instruments, such as a telescope, a microscope, a camera, a human eye, etc. are provided, allowing for a simple representation of the ray trace in all these instruments, as can be seen in Fig. 1 (a) and (b). These basic experiments illustrate in a very visual way optical phenomena such as spherical aberration, which is not so easy to imagine.

Light-matter interaction is shown in a water display. Six ultrasonic atomizers spray water, which is conducted to a pyramidal setup. There, six ventilators aspire the water and create a sheet of condensed 
droplets, where an image is projected. This device is turned on and off several times to show how the image is seen at the screen only when the light interacts with matter (Fig. 1 (c)).

Luminescence experiments represent the link between Geometrical and Physical Optics: they can still see the ray trace of the laser in the liquid (relating again with light-matter interaction), but also a completely different phenomenon, luminescence. The experiment is carried out employing pure olive oil, where a green laser displays red luminescence, and an aqueous solution created by immersing the core of a fluorescent highlighter (Fig. 1 (d)). This phenomenon in pure olive oil, employed as a quality tester in the olive oil industry, is explained to the students to illustrate how science is present in our daily life.

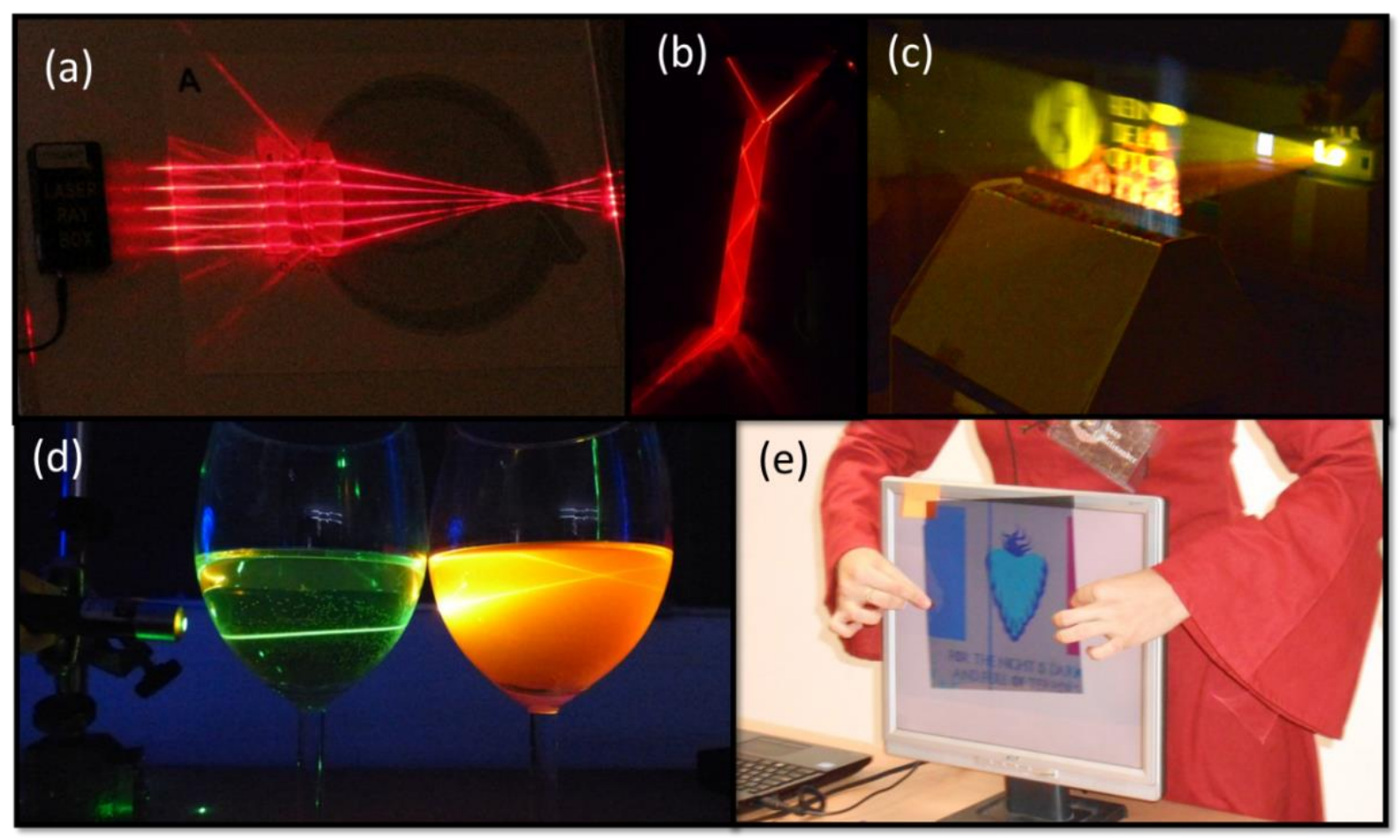

Fig.1. Several experiments included in Game of Physics: House Baratheon. (a) Ray trace with a divergent and convergent lenses, simulating light in a human eye. (b) Total reflection in a glass, the basis of optical fibre. (c) Water display: light-matter interaction.

(d) Luminescence of olive oil and of an aqueous solution when irradiated with a laser beam. (e) Polarization: by removing the polarizing film from a computer screen, one can show the linear polarization of light.

\section{2.b. Light from Other Worlds}

Light from Other Worlds [12] has a futuristic atmosphere, and can be presented both in a workshop format, with small groups of 15-20 people, or in a science fair format, where all experiments are arranged in a large space, separated into different sections that are repeated continuously, while attendees spend as much time as desired in each experiment. The target audience is, in principle, general public, but it can be adapted to students of all ages depending on the detail given in the explanations.

The experiments presented in this activity are greatly varied. We explain here the most important ones:

1) Hidden messages with the use of polarization: the experiment is the one shown in Fig. 1 (e). The linear polarizer of a computer monitor has been removed; therefore no image can be seen unless the polarizer is placed again in front of the monitor. Moreover, the original colour is only recovered for a certain orientation of the polarizer, because the RGB system assumes a certain polarization direction, and the rotation of the polarizer changes the colour of the image. Besides, a second polarizer hides the image when its axis is placed at a $90^{\circ}$ angle with respect to the axis of the first one, while having almost no effect when their axes are parallel. The concepts of linear polarization, rotation of the polarization and total extinction are explained by this simple experiment. Circular polarization is illustrated with the aid of 3D glasses used in 3D cinema, whose crystals are circular polarizers. Two volunteers put on the 
glasses and, looking in each other's eyes, wink using alternatively each of their eyes, noticing that one of their colleague's crystals darkens while the other one does not. This introduces what a $\lambda / 4$ plate is and its effect when two of them are placed successively with different orientations.

2) Luminescence in minerals, living organisms, chemical reactions, etc.: these phenomena are shown by illuminating with UV light different materials, such as minerals (calcite, fluorite and cerussite) Fig. 2(a), sea-algae (pyrocystis fusiformis), and luminescent paints (Fig. 2 (b)). It is also explained that a fluorescent lamp works analogously, i.e., by the incidence on the inner coating of the lamp of the UV light emitted by the low-pressure gas excited by an electric field (Fig. 2 (d)).

3) Laser puzzles: emulating the classical spies' movies, two different puzzles are proposed. In Fig 2. (c) a laser Khet game is displayed: the kids should guide a laser beam from one point of the table to the opposite by means of an assemble of mirrors and beam splitters. This game is an attractive way to teach how to manipulate light. Fig 2. (e) depicts a laser maze: the public must go through the maze without touching the laser beams to avoid setting off the alarm. Artificial fog needs to be created for the beams to become visible. This interactive experiment introduces the light sensors while emphasizing the lightmatter interaction idea in both the sensors and the fog.

4) Colour and visual perception: to illustrate how we perceive colour, we illuminate a small room with light coming from sodium spectroscopic lamps. The spectrum of sodium is severely discrete, emitting only few colours (mainly yellow) in the visible range. This source of illumination gives the impression that we see in "black and white", because colours like red or blue are perceived as grey.

5) Atmospheric Optics: a quite simple experiment is performed to show how turbulence in the atmosphere contributes to diffuse radiation. A laser beam is passed through a glass with water, seeing clearly the ray tracing. Little by little, sugar is added to water, and people can see how the ray tracing becomes more and more diffuse, establishing an analogy with the presence of aerosol particles in the atmosphere.

6) Holography: A real hologram fabricated with a He-Ne laser in a holographic plate is shown (Fig 2 (f)). A brief explanation of the fabrication of holograms and the applications of holography is given, focusing on the key point that the hologram is only retrieved when the same conditions as during fabrication are recovered. In addition, we teach kids how to construct an optical illusion resembling a hologram with four acetate sheets and a mobile phone (Fig 2 (g)). It is important to notice that even though this optical illusion gives the impression of a 3D image, it is created by projection of four regular images, without phase information.

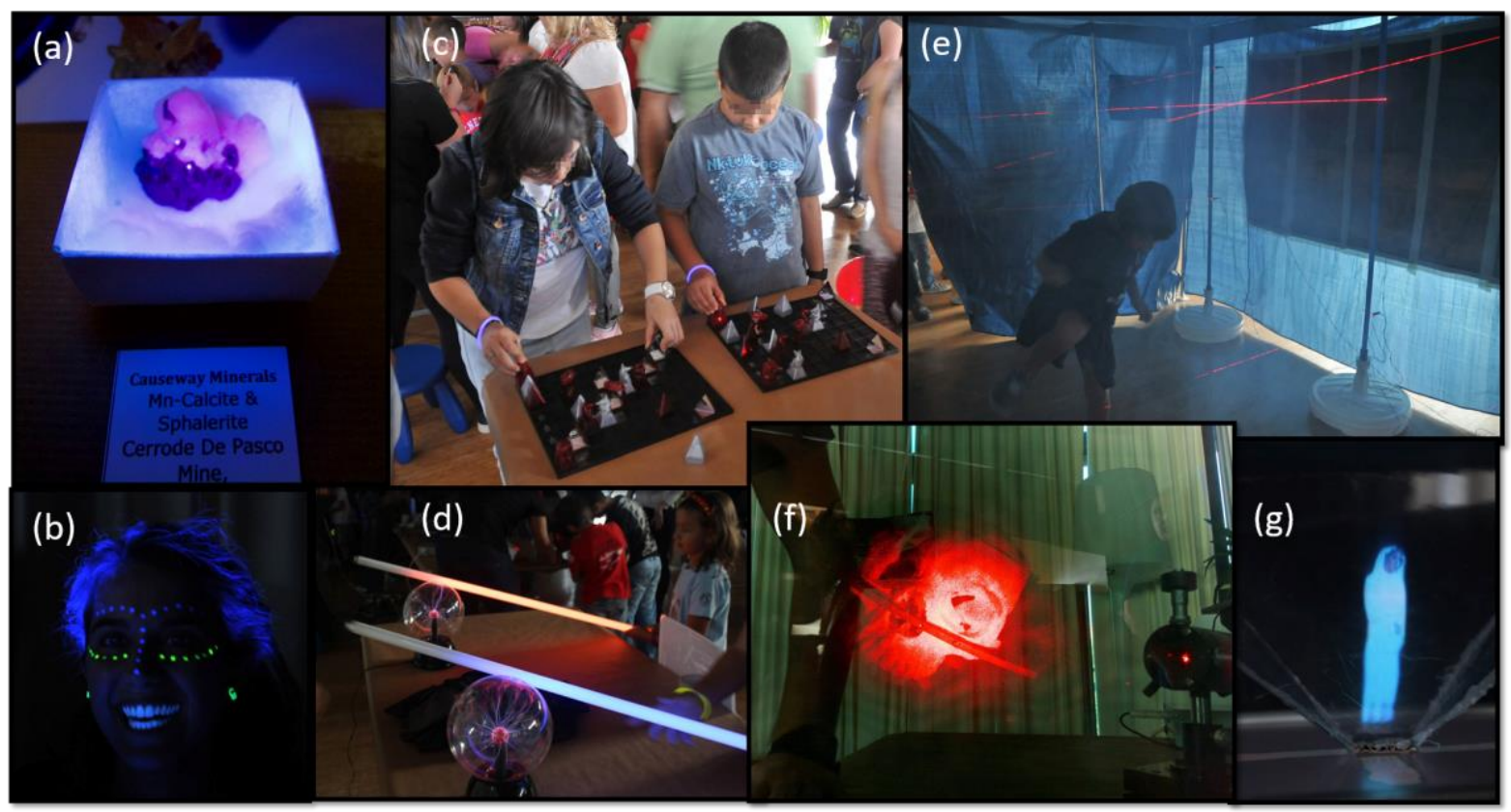

Fig.2. Photographs from Light from Other Worlds. (a) Mineral showing fluorescence when illuminated with ultraviolet light. (b) Fluorescent and phosphorescent paints illuminated by ultraviolet light. (c) Laser Khet. The participants need to reach a photosensor with a laser. (d) Fluorescent lamps being switched on due to the potential difference created between a plasma ball at one end and the participant holding the other end. (e) Laser maze: The participants are requested to cross the maze without interrupting the laser path. (f) Hologram. (g) False hologram created by projection on four acetate sheets. 


\section{2.c. Stereoptics: a 3D adventure}

This activity is designed for general public as a workshop, with small groups of 15-20 people. The aim of this workshop is to explain how 3D technology works. We begin by explaining the concept of stereoscopic vision. Our eyes perceive the same visual stimuli but with a fixed separation. The human brain is capable of combining both images in one, adding the in-depth effect. To check this idea experimentally, we perform the Pulfrich pendulum experiment [13], in which an object that oscillates in a plane seems to follow a nonplanar motion if a neutral-density filter (optical element that reduces the intensity of all the wavelengths equally) is placed in front of one of our eyes. Afterwards, the concepts of linear and circular polarization are explained (with the aid of the polarization experiment described in section 2.b) in order to understand the operation of 3D cinema, where two images are projected, with opposite circular polarizations, Fig. 3 (b). At the beginning of the workshop, a system of two slightly separated cameras takes photographs and records a small video of the attendees, Fig. 3 (a). These images and videos are processed during the workshop using 3D technology and are shown at the end as in a 3D cinema by handling out polarized glasses to the attendees, Fig. 3 (c).

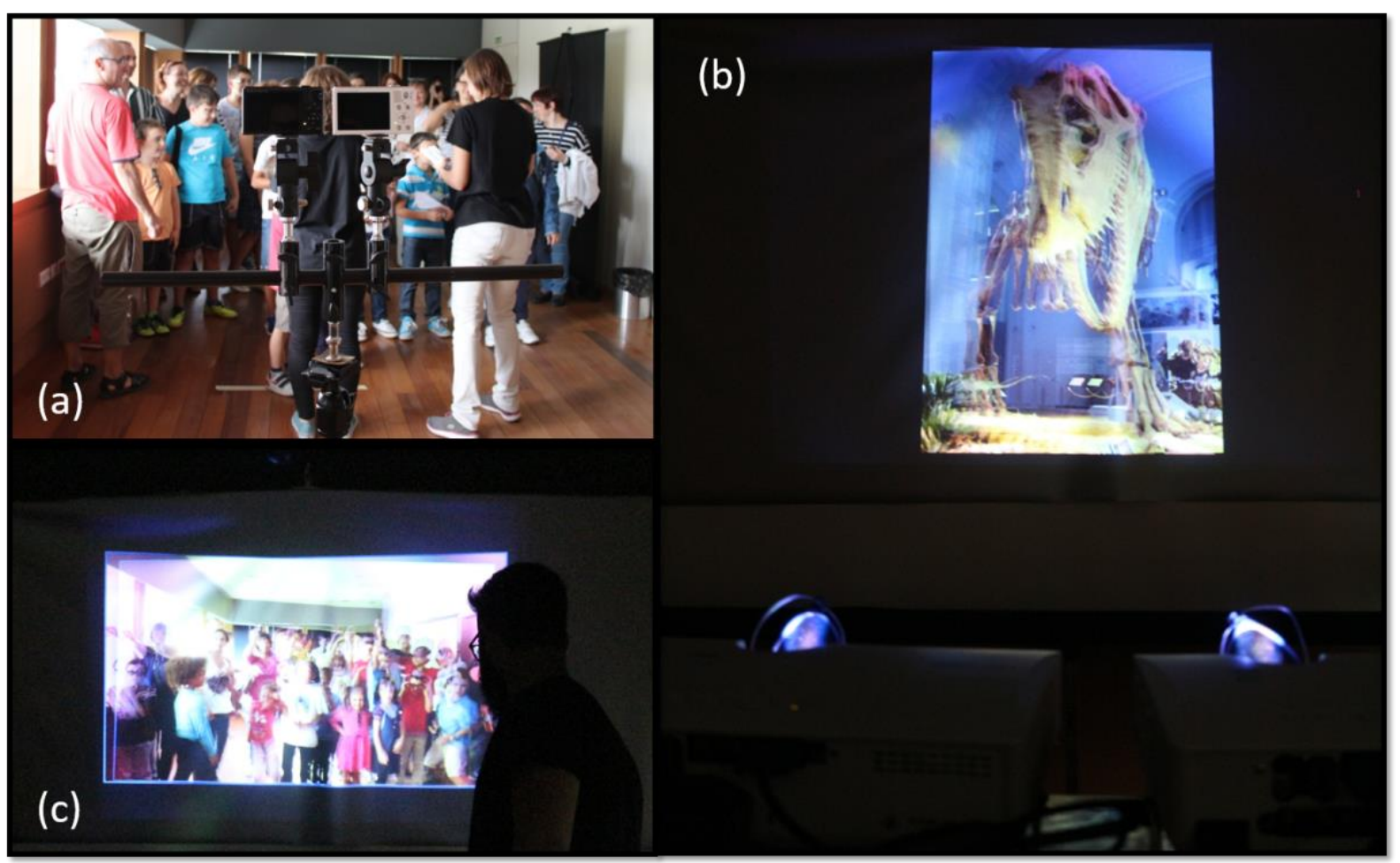

Fig.3. Images from Stereoptics. (a) Recording system. (b) 3D image created by two projectors, each one with a circular polarization (c) 3D video recorded during the workshop. Attendees are given circular polarization glasses to experience the 3D effect.

\section{2.d. Dungeons \& Photons}

This science fair format activity for the general public is one of the most recently developed, and it has been carried out for the first time in the European Researchers Night 2019 at the Science Museum of Valladolid. One of the main attractions of this event is an escape room (see Fig. 4), in which each group (5-8 people) must help a scientist who got trapped when entering a laboratory with the intention of stealing ideas from another colleague. Once the alarm goes off, they have 10 minutes before the lab notebook disintegrates. Although people of almost all ages and levels can understand and enjoy the activity (as it happens with most escape rooms), we find it more appropriate for children, as they are the kind of public which involves most in the story, in a way that makes them absorb the information without too much effort.

The first clue is in a closed jar, which contains a 'toxic' liquid, and therefore cannot be opened. However, the clue 'to empty, fill me' indicates that it is a Pythagorean cup [14], which empties when the height of the liquid it contains exceeds a certain level. Inside this cup, once emptied, there is one lens belonging to a set of $3 \mathrm{D}$ glasses. The second lens and the mount of the glasses are inside a water tank, along with water gel 
balls, which absorb great amounts of water and are invisible when immersed in the tank. This fact is used to explain refraction and the concept of refractive index and Snell's law. Once the glasses are fully assembled, the kids are asked to look at an apparently blank screen. Actually, it is a monitor from which the polarizing filter has been removed, serving as an excuse to explain the concept of polarization (see above). Using the 3D glasses, they are able to recover the image. On the screen, a laser pointer and other glasses are shown, displaying the last clue to exit the room.

The laser pointer is in a closed box with a six-letter code, Fig. 4 (b). All the letters for the code are written on the walls with fluorescent paint that needs to be illuminated with ultraviolet flashlights. This works as a way to explain the phenomenon of fluorescence (see Section 2.b). When the code (NEWTON) is introduced, the box is opened, and it contains three different targets for the laser, which are actually small diffraction grids, Fig. 4 (a) and (c). One of them shows the word 'OSA' when it is illuminated with the laser; this is the final key to exit the laboratory. The set-up of the escape room is presented in Fig. 4 (d).

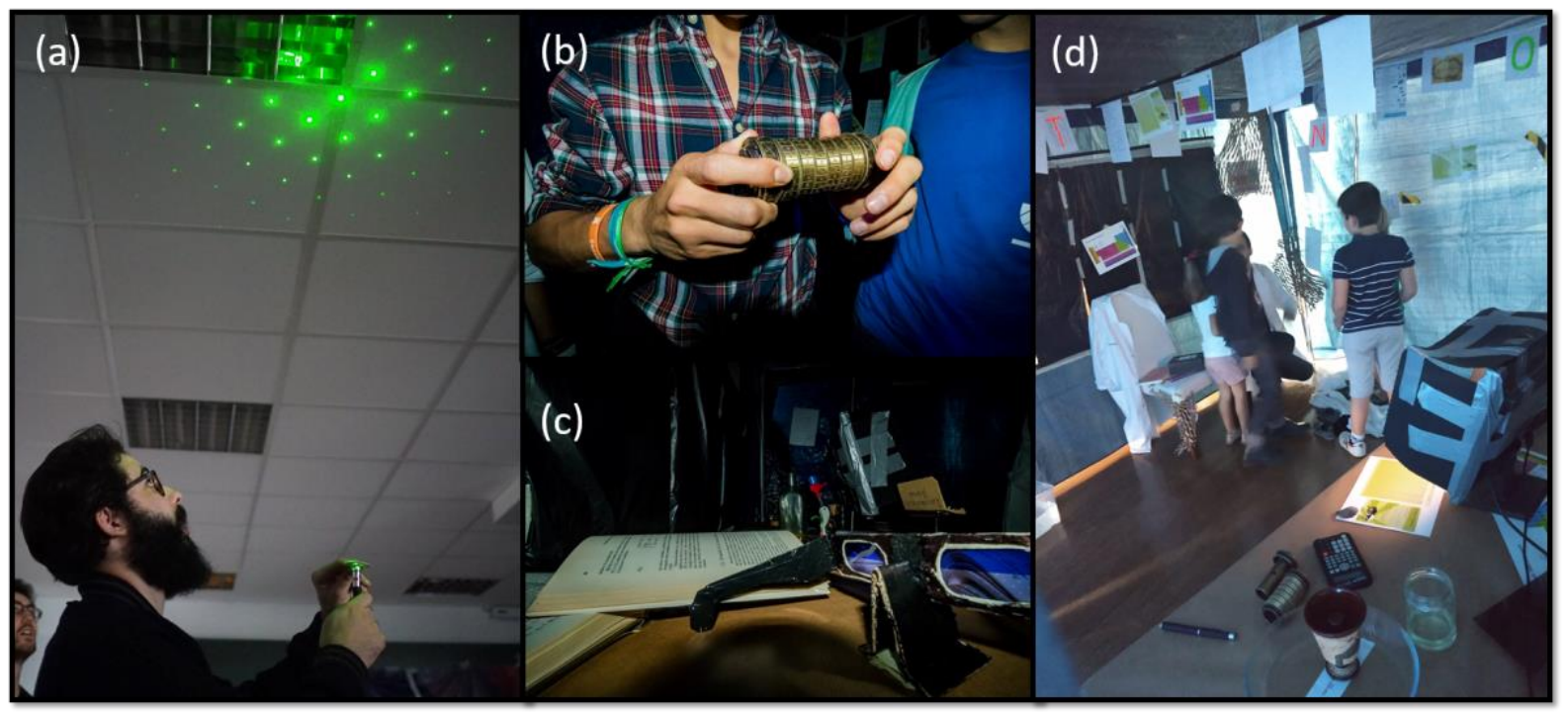

Fig.4. Images from Dungeons and Photons (a) Diffraction pattern. (b) Cryptex locked with the word NEWTON, written on the walls with fluorescent paint. (c) Diffraction grids (d) Pictures of the escape room.

\section{2.e. Scary Physics}

Scary Physics [15] is an interactive theatrical play, where the scariest side of Physics is shown. It is aimed at a general public, but was especially developed for children attending primary school. As any good terror story, it begins with a strong storm at night, which compels five Physics students to seek shelter in a seemingly abandoned house.

There, they will try to survive the tortures planned for them by terrifying villains like Jigsaw, Dr. Frankenstein, Joker and an evil sorcerer. Some of the experiments our teenagers will have to undergo will be as brutal as a bed of nails, walking on broken glass, holding fire with their own hands... but Optics is also present in this malevolent house. Teenagers will have to see how villains test their intellect with experiments based on luminescence (Fig. 5 (a), (e), (f), and (g)), learn how light interacts with matter in a Pepper's ghost experiment [16] (Fig. 5 (b)), discover the secrets of reflection and transmission (Fig. 5(c)), and how we can light up a fluorescent lamp without wires (Fig. 5 (d)). All the experiments are explained during the play, by the adolescents or the antagonist. The plot flow is not interrupted because the characters interact with the experiments and explanations. Would you dare to enter? 


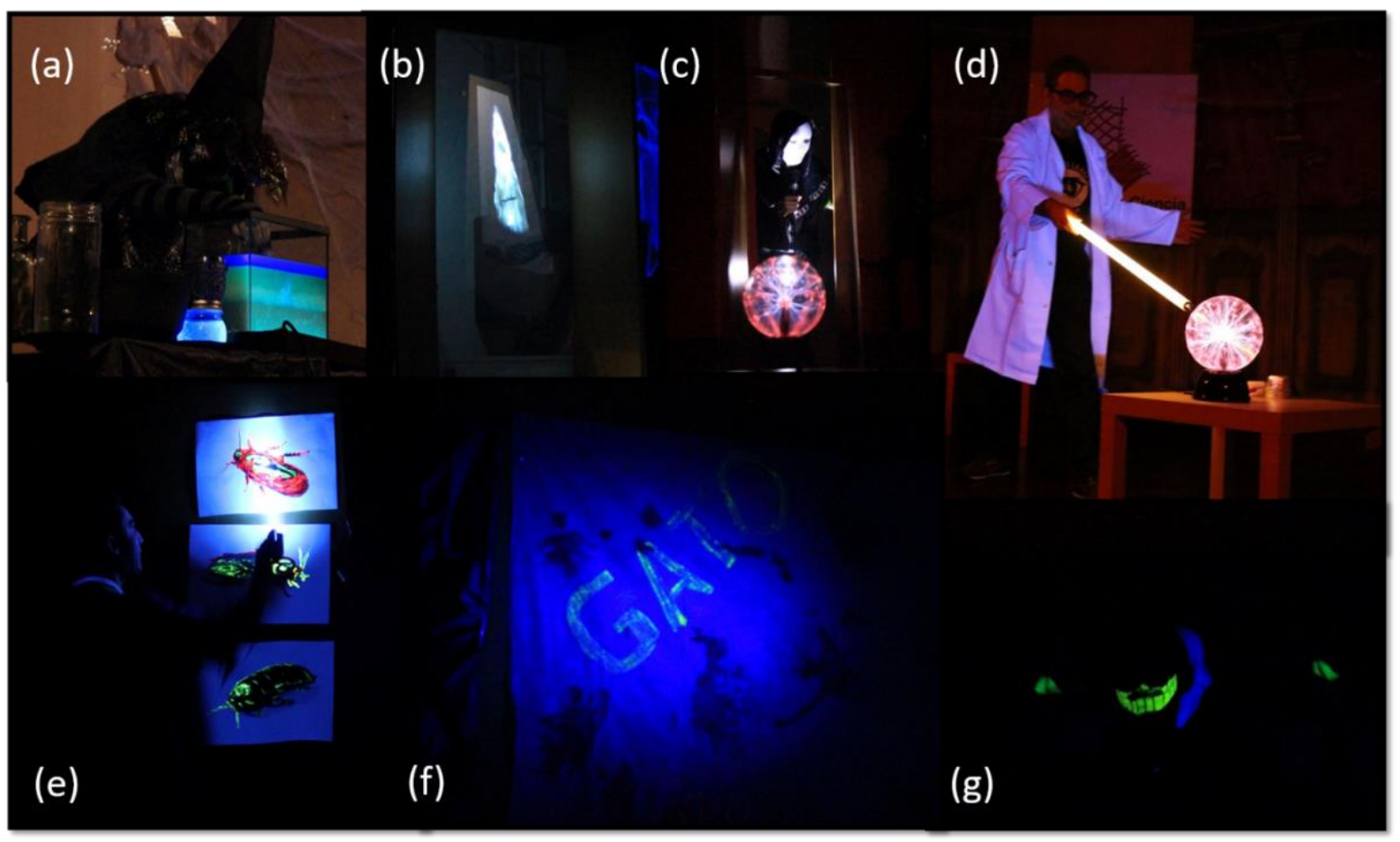

Fig.5. Images from Scary Physics. (a) Liquid tower, to show the concept of density. (b) Pepper's ghost effect. (c) Reflection (not shown) and transmission of light. (d) Lighting up a fluorescent bulb with a plasma ball. (e), (f), and (g) Luminescence experiments.

\section{Conclusions}

We have presented the outreach activities related to Optics and Photonics carried out by the non-profit association Physics League. By creating an entertaining atmosphere based on TV shows and movies, we have shown five different formats in which to showcase multiple optical experiments. The key points to be stressed regarding this approach are: 1) the interaction of the public with the experiments, in contrast with mere demonstration; 2) the setting of the experiments in a universe or story, real or fictional, which the public might feel identified with or attracted to; and 3) the use of uncommon staging such as theatrical plays or escape rooms. The aforementioned activities have been carried out in front of more than 10,000 people as a whole, with very good acceptance and evaluation. Given this success, we hope this article will encourage other outreach associations to carry out some activities in similar formats as described above, or inspire them to develop new formats and approaches.

\section{Acknowledgements}

The authors thank the European Physical Society and its Young Minds project, the Optical Society of America, the American Physical Society, the Valladolid Deputation for the funding received, and the Science Faculty of the University of Valladolid for the provided facilities. We also thank the Science Museum of Valladolid for the support in our joint activities. Thanks are also due to all the members of the Physics League for their valuable contributions and the time invested in these activities. 